\title{
Conocimientos económicos y financieros de los estudiantes de último grado de secundaria en Santander, Colombia Economic and financial knowledge of high school seniors in Santander, Colombia
}

\author{
Silvia Nathalia Núñez Rueda ${ }^{1}$, Luis Alejandro Palacio García ${ }^{2}$ \\ snanurue@correo.uis.edu.co ${ }^{1}$, lpalagar@uis.edu.co ${ }^{2}$ \\ Universidad Industrial de Santander \\ Bucaramanga, Colombia
}

\begin{abstract}
Resumen- Para todas las sociedades, resulta fundamental formar una cultura de ahorro para administrar sus presupuestos de forma responsable, pues un grupo social que no sabe cómo planear sus ingresos y gastos, no puede mejorar su bienestar social. En este contexto, la Educación Económica y Financiera -EEF- es una opción para formar ciudadanos responsables de sus decisiones, pues un comportamiento financiero irresponsable implica la aparición de deudas, en adultos y jóvenes. Para Colombia, los resultados del conocimiento de los estudiantes en temas de EEF son negativos. Entre los países evaluados en la prueba PISA 2018, el país obtuvo el puesto 58 de 79. Por tanto, se realizó un diagnóstico con una muestra de 1.016 estudiantes de último grado de secundaria de 13 instituciones educativas de Santander, para conocer sus habilidades y conocimientos económicos y financieros. Además, se realizaron 13 grupos focales con profesores para conocer la planeación didáctica de estas instituciones. A partir de los resultados se evidencian debilidades en la planeación didáctica en temas de EEF en la mayoría de las instituciones, lo cual se traduce en algunas deficiencias en las habilidades y los conocimientos económicos y financieros de los estudiantes.
\end{abstract}

Palabras clave: educación; necesidades educacionales; desarrollo; competencias del docente y finanzas.

\begin{abstract}
To societies is essential to form a culture of savings to manage their budgets in a responsible way, since a social group that does not know how to plan its income and expenses cannot improve its social well-being. In this context, Economic and Financial Education EFE- is an option to train citizens responsible for their decisions, since irresponsible financial behavior implies the appearance of debts, in adults and young people. For Colombia, the results of students knowledge of EFE issues are negative. Among the countries evaluated in the PISA 2018 test, the country ranked 58 out of 79. Therefore, was made a diagnosis with a sample of 1,016 high school seniors from 13 educational institutions in Santander, to learn about their economic and financial skills and knowledge. In addition, 13 focus groups were held with teachers to learn about the didactic planning of these institutions in the teaching of EFE. From the results, weaknesses are evidenced in the didactic planning on EFE issues in most of the institutions, which translates into some deficiencies in the economic and financial skills and knowledge of the students.
\end{abstract}

Keywords: education; education needs; development, teacher competencies and finance

\section{INTRODUCCIÓN}

Hoy en día tomar una decisión financiera acertada es difícil, pues exige que los individuos comprendan diferentes conceptos como el costo futuro del dinero, las ganancias, las pérdidas y, además, resistan la tentación de consumir demasiado sin necesidad alguna (Banco Mundial, 2015). No obstante, desde hace algunos años la facilidad para encontrar en el mercado productos y servicios financieros ha aumentado en número y en complejidad (Departamento Nacional de Planeación, 2019), pues gracias a los cambios tecnológicos, se han gestado nuevas opciones para la inclusión financiera, pero también ello ha asociado nuevos riesgos producto de los bajos niveles de conocimientos, competencias y habilidades en EEF por parte de la población, lo cual sumado a estos tiempos de "modernidad líquida" donde las estrategias de proyectos de vida a largo plazo se ven socavadas por las expectativas de corto plazo (Bauman, 2012), genera un aumento en la obligación de los gobiernos para formar en estas temáticas a sus ciudadanas y ciudadanos, y de este modo lograr que la EEF influencie de forma positiva la toma de decisiones en la población.

Teniendo en cuenta que un comportamiento financiero irresponsable y habilidades financieras deficientes implican la aparición de deudas en los adultos y los jóvenes (Amagir, Groot, Maassen, Brink \& Wilschut, 2018), se vislumbra una necesidad inmediata de incluir la EEF en la vida de los estudiantes. Respecto al tema, diversos autores coinciden que es necesario atender esta necesidad e incorporar al currículo escolar programas que permitan educar económica y financieramente a los estudiantes, iniciando desde la educación primaria, repitiendo en la educación secundaria y continuando en la universidad (Amagir et al., 2018). Es importante, de manera especial impactar en los comportamientos financieros (es decir, la habilidad, la actitud y el conocimiento) de los estudiantes, pues estos se ven afectados de forma positiva por la implementación de la educación financiera impartida a través del currículo escolar (Cordero \& Pedraja, 2019).

En general, se define EEF como el proceso por el cual los consumidores e inversores mejoran su conocimiento sobre los productos, conceptos y riesgos financieros $\mathrm{y}$, a través de información, instrucción y/o consejo objetivo, desarrollan las habilidades y confianza para adquirir una mayor conciencia de los riesgos y oportunidades para tomar decisiones informadas que permitan mejorar su bienestar (OCDE, 2005). Siguiendo el 
trabajo de revisión realizado por Gnan, Silgoner \& Weber (2007), la manera como se presenta el contenido de los programas enfocados en formar en temas de EEF, depende en gran medida de los motivos y objetivos de los distintos proveedores de educación. Por ejemplo, los bancos centrales, brindan EEF para mejorar la efectividad de la política monetaria o asegurar el buen funcionamiento de los mercados financieros (Aprea et al., 2016).

Además, de acuerdo con Valbuena, Marín y De la Hoz (2020), la EEF permite a los individuos aprovechar los servicios y productos financieros de una mejor manera, pues brinda las competencias necesarias para resolver y analizar los desafíos financieros a los cuales se enfrentan los jóvenes a diario. Además, conocer la forma correcta de administrar los ingresos y los gastos, tomar mejores decisiones y evitar altos niveles de endeudamiento, permitirá mejorar la calidad de vida de las personas expuestas al aprendizaje (Valbuena et al., 2020). En este sentido, hablar de EEF puede reconocerse como un factor efectivo para reducir la exclusión social y desarrollar un sistema financiero sólido (Raccanello, Kristiano, Herrera y Guzmán, 2014).

Adicionalmente, debe tenerse en cuenta que la EEF va más allá de solo conocimientos. Según Compen, De Witte y Schelfhout (2018), la EEF incluye también la actitud financiera (voluntad de ahorrar dinero a largo plazo) y el comportamiento financiero (tener un presupuesto familiar). Esto implica que la comprensión objetiva de contenidos financieros es insuficiente, por lo que se requiere desarrollar procesos de pensamiento crítico que conlleven aprendizajes significativos (Danes, Rodríguez \& Brewton, 2013).

\section{CONTEXTO}

En Colombia, los resultados de las mediciones de capacidades económicas y financieras son poco favorables (Banco Mundial, 2013; Asobancaria, 2018). De acuerdo con los últimos resultados de las pruebas PISA, se observa que, entre los países evaluados, el país obtuvo el puesto 58 de 79 que se presentaron (OCDE, 2020). Lo anterior, evidencia un desconocimiento de las finanzas personales por parte de los estudiantes de educación secundaria del país.

De acuerdo con el Plan Nacional de Desarrollo para Colombia 2018 - 2022 (DNP, 2019), una de las estrategias para continuar en el avance de la inclusión financiera, es la enseñanza de la EEF con enfoque digital. En ese sentido, se han realizado varios esfuerzos para procurar una adecuada educación de los consumidores financieros del país, especialmente los más jóvenes. Además, se suman esfuerzos posteriores, como el planteado en 2012 por el Ministerio de Educación Nacional de Colombia (MEN) y la Asociación Bancaria y de Entidades Financieras de Colombia (Asobancaria), los cuales suscribieron un convenio para la implementación de un programa de Educación Económica y Financiera en los establecimientos educativos del país. A través de estos esfuerzos, se desarrolló el

Documento No. 26 “Orientaciones Pedagógicas para la Educación Económica y Financiera”. Sin embargo, a pesar de estas iniciativas, tal como lo demuestran las recientes mediciones de diferentes instituciones, los resultados en temas de EEF de los jóvenes colombianos no son muy alentadores.
Por tanto, y considerando que el método más poderoso para mejorar la EEF de los jóvenes es aumentar la cobertura de las finanzas personales en el currículo escolar (Jang, Hahn, \& Park, 2014), un grupo de investigadores de la Universidad Industrial de Santander - UIS- (Colombia) realizó un diagnóstico con una muestra a conveniencia de 1.016 estudiantes de último grado de secundaria de instituciones educativas públicas del departamento de Santander, para conocer sus habilidades y conocimientos económicos y financieros. Además, se realizaron 13 grupos focales con profesores para conocer la planeación didáctica de estas instituciones en lo referente a la enseñanza de la EEF.

A partir de los resultados se evidencian debilidades en la planeación didáctica en temas de EEF en la mayoría de las instituciones educativas, lo cual se traduce en algunas deficiencias en las habilidades y los conocimientos económicos $\mathrm{y}$ financieros de los estudiantes en Santander. Con estos resultados, durante 2021 se lleva a cabo el diseño de una herramienta gamificada (videojuego) que facilite el desarrollo de habilidades y conocimientos económicos y financieros. Con esto, se busca que los jóvenes suplan sus necesidades en la formación de temáticas asociadas a la EEF, y que derivado de ello puedan construir estilos de vida sostenibles que promuevan el desarrollo de las sociedades en las cuales participan.

A continuación, se presenta un breve resumen de la importancia de la EEF para la vida de los jóvenes y su inclusión en el currículo escolar. En el apartado siguiente se presenta la metodología implementada para realizar el diagnóstico de las habilidades y los conocimientos económicos y financieros de los jóvenes de Santander; además, se explica de forma breve, los grupos focales realizados con los profesores de las instituciones participantes con el objetivo de identificar las metodologías, materiales y formas de evaluación utilizadas en las instituciones. Luego, se presentan los principales resultados de este diagnóstico y los grupos focales realizados para finalizar en el último apartado con la presentación de unas conclusiones surgidas a partir del trabajo de investigación realizado.

\section{DESCRIPCIÓN}

La metodología implementada para realizar el diagnóstico de las habilidades y los conocimientos en temas de EEF de las y los estudiantes participantes en Santander, incluyó el diseño de un instrumento de medición diseñado por el equipo investigador. Además, con el objeto de revisar las metodologías, los materiales y los sistemas de evaluación implementados en las instituciones, se realizaron 13 grupos focales con profesores de las instituciones participantes. A continuación, se describe el diseño del instrumento utilizado, la población participante y la metodología de grupos focales implementada.

\section{A. Diseño del instrumento de medición}

Para el diseño del instrumento de medición se usó como base el cuestionario OCDE/INFE Toolkit for measuring financial literacy and financial inclusion (OCDE, 2018). En su versión original, el instrumento permite identificar los comportamientos de los individuos sobre el manejo del dinero a corto plazo, el manejo del dinero día a día y a largo plazo, la planeación financiera, entre otros (Kempson, 2009). Para este estudio en particular se modificó el cuestionario, ajustando algunos componentes por recomendación de un grupo de 
investigadores expertos en el área de Economía, Finanzas y Educación de la Universidad Industrial de Santander. Además, se ajustó la escala de medición del instrumento, el cual maneja diferentes escalas de respuesta, dependiendo de su sección.

Una vez surtido este proceso con expertos, el instrumento fue estructurado en 41 preguntas, distribuidas en 10 componentes. Estos componentes se mencionan a continuación: i) Características personales y del hogar; ii) Información de contexto (autopercepción de conocimientos económicos y financieros); iii) Planificación de finanzas y gestión de finanzas; iv) Ahorro activo y choques financieros; v) Metas financieras; vi) Planificación financiera a largo plazo; vii) Gastos mensuales; viii) Actitudes y comportamiento; ix) Conocimiento financiero; y x) Conocimiento de productos financieros.

Antes de la aplicación del cuestionario, en marzo de 2020 se realizó una prueba piloto de forma presencial con un grupo de 220 estudiantes de último grado de secundaria de una de las instituciones participantes. Posterior a esta prueba, fueron identificadas algunas falencias en el lenguaje y opciones de respuesta, que fueron corregidas sin inconveniente. Una vez ajustado el instrumento de medición, entre abril y mayo de 2020, los estudiantes de último grado de secundaria participantes, con el permiso previo de su tutor, diligenciaron el cuestionario de forma virtual. Para el tratamiento y análisis de los datos el equipo de proyecto implementó el uso del software de analítica Tableau.

\section{B. Población participante}

Para el estudio se seleccionaron estudiantes de último grado de secundaria de 13 instituciones educativas públicas de Santander. De acuerdo con lo identificado, un 54\% de los participantes eran mujeres y un $46 \%$ hombres. Respecto a la distribución por edad se encuentró que un 52\% tienen 16 años y un $25 \%$ cuenta con 17 años. Se resalta que el porcentaje de participación de estudiantes mayores de edad (18 años o más) es de $11 \%$. En lo referente a la distribución por estrato1, se observó que la mayoría de los estudiantes se ubican en el estrato dos con $32 \%$. En segundo lugar, se ubican los participantes de estrato tres con $29 \%$. Se resalta que existe una baja concentración de participantes en los estratos cinco y seis, con solo el $0.5 \%$ del total.

Adicionalmente, se evidenció que la mayoría de los participantes ubicaron los ingresos de sus hogares en pesos colombianos por debajo de $\$ 1.000 .000$ (275 USD) al mes, con un $33 \%$. En segundo lugar, se tiene el rango de $\$ 1.000 .000$ a $\$ 2.000 .0000$ (275 USD - 551 USD) con un 25\%. Para el rango entre $\$ 2.000 .000$ y $\$ 3.000 .000$ (551 USD - 827 USD), se tiene un $11 \%$, y en el rango entre $\$ 3.000 .000$ y $\$ 4.000 .000(827$ USD- 1.103 USD) un $4 \%$. Sin embargo, es de notar que un $27 \%$ manifiesta no saber el monto de los ingresos de sus hogares.

\section{Grupos focales con los directivos y los profesores de las instituciones educativas}

Con las 13 instituciones educativas participantes se realizaron grupos focales con cada una para indagar por las opiniones y las percepciones de los profesores y los directivos sobre las metodologías de enseñanza, los materiales utilizados en las clases relacionadas con temáticas de EEF y la forma de evaluación para conocer la planeación didáctica que se desarrolla en cada institución. Estas sesiones estuvieron lideradas por un moderador que coordinó la reunión con una guía de preguntas orientadoras preparadas previamente
(Aravena et al., 2006). Como asistentes participaron investigadores de la Universidad Industrial de Santander, directivos y profesores de las instituciones. Al final del ejercicio con los grupos focales realizados, se contabilizó la participación de 55 profesores y directivos.

\section{RESULTADOS}

A continuación, se presentan algunos de los resultados más relevantes obtenidos en lo referente al diagnóstico realizado mediante el instrumento de medición diseñado y los grupos focales realizados.

\section{A. Resultados instrumento de medición}

Conocimientos económicos y financieros. Para identificar los conocimientos económicos y financieros, se realizaron 5 preguntas en escala de menor a mayor complejidad para conocer la aplicabilidad en los cálculos matemáticos que realizan los estudiantes para resolver problemas sencillos relacionados con términos como tasas de interés, prestamos, inflación e interés. En lo referente a las preguntas uno y tres los porcentajes de acierto en general fueron iguales o mayores al $88 \%$. Para estas preguntas los estudiantes debían estar en la capacidad de entender el concepto de préstamo e interés. La pregunta de más bajo acierto corresponde a la pregunta dos con un $31 \%$. Esta pregunta específica, involucraba el conocimiento del término inflación para su respuesta correcta.

En la pregunta cuatro, relacionada con el cálculo de una tasa de interés simple se obtuvo un acierto del $33 \%$, el cual es bajo, teniendo en cuenta que los estudiantes de último grado de secundaria de Colombia deben estar en capacidad de responder este tipo de problemas, de acuerdo con las orientaciones estipuladas por el Ministerio de Educación Nacional.

Conocimiento de productos financieros. Se identificó en general que los estudiantes tienen un bajo conocimiento de productos financieros de deuda e inversión. En general, el producto de deuda más conocido es la Tarjeta de crédito con un 95\% y el menos conocido el Crédito de libranza con un 16\%. Para los productos financieros de inversión, se observó que el más conocido es la Cuenta de ahorro con un 94\% y el menos conocido el Certificado de Depósito de Término con un $23 \%$.

Autopercepción de conocimientos económicos y financieros y clases de EEF en las instituciones educativas. Se observó que un $25 \%$ de los participantes manifiesta tener conocimientos "Muy bajos" y "Bajos" en lo referido a EEF. La opción con menor porcentaje se ubica en el nivel "Más alto" con un $1 \%$. En lo referente a la pregunta si los participantes reciben o no clases de EEF en sus instituciones educativas, se encuentra que un 49\% manifestó en general recibir estas clases.

Planificación y gestión de finanzas. En lo referente a las acciones que realizan las y los estudiantes para la planificación y gestión de sus finanzas, se observa que un 53\% manifiesta mantener el dinero para los gastos fijos separado del dinero que gasta día a día; un $44 \%$ hace un plan para administrar sus ingresos y gastos; un 43\% toma nota de sus gastos; y un $9 \%$ usa una aplicación bancaria o una herramienta de administración de dinero.

Ahorro activo y choques financieros. Se observa que las y los estudiantes, en un $89 \%$, prefieren el ahorro de dinero en efectivo en una alcancía o en su billetera. En lo referido a ahorrar dinero en una cuenta de ahorro se tiene que un $21 \%$ ahorran de esta forma. Lo anterior, evidencia que existe una 
mayor propensión al ahorro de dinero en efectivo a través de canales informales.

Metas financieras. Se identificó que un $88 \%$ de los participantes manifestaron tener algún tipo de meta financiera a corto, mediano o largo plazo. La meta financiera más popular, con un $36 \%$ fue pagar los estudios universitarios.

\section{B. Resultados de los grupos focales realizados con directivos y docentes}

En lo referente a las estrategias didácticas, los materiales y los métodos de evaluación implementados por las 13 instituciones educativas participantes, en general se observa un patrón similar en el desconocimiento y aplicabilidad de metodologías prácticas y efectivas para la formación de conocimientos y habilidades relacionadas con la EEF.

Por ejemplo, se identifica que en la mayoría de las instituciones la EEF se concibe solo a través de las áreas de matemáticas y cálculo, no se evidencia otra asignatura que trate el tema. En general la metodología más popular es la implementación de ejercicios prácticos en Microsoft Excel sobre el cálculo de tasas de interés y otros conceptos que requieran el uso de algún tipo de fórmula que ofrece esta herramienta.

Para la enseñanza de temáticas relacionadas con el ahorro, se observó que algunas instituciones promueven el ahorro de recursos naturales y dinero mediante el diseño y construcción de una alcancía. Lo anterior, promueve el ahorro de dinero en efectivo entre los estudiantes para alcanzar sus diferentes metas personales, especialmente en los grados de primaria. Solo dos instituciones de las trece participantes realizan eventos propios como congresos o simposios que motiven a las y los estudiantes en el aprendizaje de temas relacionados con EEF.

En lo concerniente a los materiales que apoyan la formación de los estudiantes, se observó que menos de la mitad utiliza cartillas de apoyo suministradas por instituciones aliadas como bancos privados. Casi ninguna tiene en cuenta en su planeación los documentos oficiales del Ministerio de Educación de Colombia. En general, la constante en estos materiales son los libros de matemáticas, en especial, los que contengan problemas asociados al cálculo de tasas de interés y el ahorro. Algunas instituciones implementan materiales didácticos como juegos a través de casinos simulados, ruletas y dominós.

Por último, en lo referente a la evaluación de los procesos que adelantan las instituciones educativas para revisar la formación de conocimientos y habilidades relacionadas con el área de EEF, es una constante que se realicen evaluaciones sumativas de los temas tratados en el área de matemáticas, pero sin discriminar específicamente por temas de EEF. Es decir, no se tiene prevista una evaluación en las instituciones para revisar la formación de estos conocimientos en los estudiantes. Solo dos instituciones educativas manifestaron realizar evaluaciones cualitativas a través de encuestas. Además, promueven una autoevaluación del proceso con el objetivo que el estudiante identifique sus fallas y pueda corregirlas.

\section{CONCLUSIONES}

A continuación, se presentan las conclusiones del estudio realizado a partir de un análisis integrado de las dos fuentes de información recolectadas: i) resultados del diagnóstico de las y los estudiantes y ii) conclusiones de los grupos focales con los directivos y los profesores de las trece instituciones educativas.

A partir de este análisis se identificó que los participantes que auto reportan altos niveles de conocimientos en temas económicos y financieros manifiestan recibir clases de EEF en sus instituciones. Lo anterior, se resalta como algo positivo, pues resulta fundamental incluir la EEF como uno de los métodos más valiosos para promover el desarrollo de los jóvenes en temas de finanzas personales (Jang, Hahn, \& Park, 2014). Independiente del enfoque que decidan emplear, estos programas deben ser diseñados para satisfacer las necesidades y el nivel de conocimientos financieros de la población para generar cambios en su bienestar financiero (OCDE, 2005).

Además, entre conductas positivas se identifica que la mayoría de las y los estudiantes manifiestan tener una meta financiera a corto, mediano o largo plazo. Además, se evidenció que las y los participantes tienen una propensión al ahorro de dinero en efectivo a través de diferentes canales informales, tales como ahorrar en alcancía o la billetera.

Respecto a las debilidades en los conocimientos económicos $\mathrm{y}$ financieros de los estudiantes participantes, se observa que en el instrumento de medición las preguntas que involucraron el uso de términos como inflación o tasa de interés simple corresponden a las de más bajo acierto. Igualmente, se observó que en general los participantes no conocen los productos financieros básicos de deuda e inversión. Por el contrario, evidencian un mayor conocimiento de productos financieros informales como son los prestamistas o las casas de empeño.

Además, resulta preocupante que las preguntas de conocimientos económicos y financieros que involucraron el uso de operaciones matemáticas combinadas para llegar a su respuesta, obtuvieron un bajo nivel de acierto. Lo anterior, está unido a la ausencia de conocimientos en temas como el riesgo y su relación con la diversificación de inversiones o mercados de acciones.

A partir de lo anterior, es importante implementar proyectos a corto plazo en las instituciones que permitan mejorar y reforzar el conocimiento relacionado con temas como los nuevos productos financieros, los canales de ahorro formales, las estrategias de planeación de gastos, las opciones para la obtención de un crédito en el mediano y largo plazo, entre otras. Ello resulta fundamental, pues los jóvenes se enfrentan a diario a complejos productos que si no son comprendidos en sus potencialidades y riesgos pueden conllevar a un deterioro significativo de su calidad de vida y de su futuro (Vera, 2016). Por esto se hace necesario ofrecer a los jóvenes las herramientas para su comprensión y posterior administración (Lusardi \& Mitchell, 2014).

Ahora bien, los resultados anteriores evidencian debilidades en la planeación didáctica en temas de EEF en la mayoría de las instituciones, lo cual se traduce en algunas deficiencias en las habilidades y los conocimientos económicos y financieros de los estudiantes. Lo anterior, constituyó la base para la construcción de una herramienta gamificada (videojuego) llamado "FINATIC" una ciudad sostenible, que permita el desarrollo de habilidades económicas y financieras, que suplan las necesidades de los jóvenes del departamento de Santander (Colombia) en temas de EEF, y que además, puedan apoyar a las instituciones educativas en la formación en esta área. 
Esta herramienta se encuentra en construcción en la actualidad, y se espera que esta estrategia basada en juego permita aumentar la motivación de las y los estudiantes por estos temas (Acosta-Medina, Torres-Barreto, ÁlvarezMelgarejo \& Paba- Medina, 2020) y, además, sirva como una herramienta didáctica para dinamizar las actividades planeadas por los profesores en los temas relacionados con EEF. Los contenidos incluidos en "FINATIC: una ciudad sostenible", se seleccionaron de acuerdo con las orientaciones de EEF propuestas por el Ministerio de Educación de Colombia y las necesidades educativas identificadas en los estudiantes. Estos contenidos fueron construidos de tal manera que, conforme se superan los niveles del videojuego aumente el nivel de dificultad de las temáticas. Además, se planteó como un videojuego de construcción y gestión de recursos de tipo plataforma web en el que el jugador se encarga de controlar y administrar los recursos disponibles, a la vez que afronta diferentes misiones nivel a nivel.

Como retos futuros, se plantea ampliar su cobertura hacia educación básica y educación superior, en esta última, por ejemplo, como apoyo en la formación de áreas como la administración, las finanzas o la economía. Además, resulta interesante para el equipo de investigación profundizar en el estudio estadístico de las diferencias de los conocimientos económicos y financieros en los centros educativos con más y menor formación, así como en función de los estratos en general.

\section{REFERENCIAS}

Acosta-Medina, J. K., Torres-Barreto, M. L., ÁlvarezMelgarejo, M., \& Paba-Medina, M. C. (2020). Gamificación en el ámbito educativo: Un análisis bibliométrico. I+ D Revista de Investigaciones, 15 (1), 28-36.

Amagir, A., Groot, W., Maassen van den Brink, H., \& Wilschut,

(2018). A review of financial-literacy education programs for children and adolescents. Citizenship, Social and Economics Education, 17(1), 56-80. DOI: https://doi.org/10.1177/2047173417719555.

Aprea, C., Wuttke, E., Breuer, K., Koh, NK, Davies, P., Greimel-Fuhrmann, B. y Lopus, JS (Eds.). (2016). International Handbook of Financial Literacy. Singapur: Springer.

Aravena, M., Kimelman, E., Micheli, B., Torrealba, R., \& Zúñiga, J. (2006). Investigación educativa I.

Asobancaria (2018). Índice de educación financiera en los colegios de Asobancaria. Recuperado de: $\quad$ https://www.asobancaria.com/wpcontent/uploads/2018/03/1127C-05- 03-2018.pdf

Banco Mundial (2013). Capacidades financieras en Colombia: resultados de la encuesta nacional sobre comportamientos, actitudes y conocimientos financieros. Recuperado de: http://www.bancomundial.org/content/dam/Worldbank/d oc ument/LAC/Capacidades $\% 20$ Financieras $\% 20$ en $\% 20$ Colombia.pdf

Banco Mundial. (2015). Informe sobre el desarrollo mundial 2015: mente, sociedad y conducta - panorama general: Mind, Society, and Behavior. Washington. Recuperado de:

https://openknowledge.worldbank.org/handle/10986/205 97 License: CC BY 3.0 IGO.

Bauman, Z. (2012). Liquid Modernity (2012th ed.). Polity Press.

Compen, B., De Witte, K., Schelfhout, W. (2018). The role of teacher professional development in financial literacy education: A systematic literature review. Educational Research Review, 26, 16-31. DOI: https://doi.org/10.1016/j.edurev.2018.12.001.

Cordero, J. M., \& Pedraja, F. (2019). The effect of financial education training on the financial literacy of Spanish students in PISA. Applied Economics, 51(16), 16791693. https://doi.org/10.1080/00036846.2018.1528336.

Danes, S., Rodriguez, M., \& Brewton, K.E. (2013). Learning Context When Studying Financial Planning in High Schools: Nesting of Student, Teacher, and Classroom Characteristics. Journal of Financial Counseling and Planning, 24, 20-36. Recuperado de: https:/www.semanticscholar.org/paper/LearningContext- When-Studying-Financial-Planning-DanesRodriguez/3a9fcdb9d22c703f8076ebb06e2fce489af3d63 7

Departamento Nacional de Planeación. (2019). Bases del Plan Nacional de Desarrollo 2018-2022.

Gnan, E., Silgoner, M. A., \& Weber, B. (2007). Economic and financial education: Concepts, goals and measurement. Monetary Policy \& the Economy Q, 3(1), 28-49. Recuperado de:

https://econpapers.repec.org/article/onboenbmp/y_3a200 7_3ai_3a3_3ab_3a2.htm.

Jang, K., Hahn, J., \& Park, H. J. (2014). Comparison of financial literacy between Korean and U.S. high school students. International Review of Economics Education, 16(PA), 22-38. DOI: https://doi.org/10.1016/j.iree.2014.07.003.

Lusardi, A., \& Mitchell, O. (2014). The economic importance of financial literacy: Theory and evidence. Journal of Economic Literature, 52(1), 5-44. DOI: https://doi.org/10.1257 / jel.52.1.5.

OCDE -Organización para la Cooperación y el Desarrollo Económicos. (2018). OCDE/INFE: Toolkit for Measuring Financial Literacy and Financial Inclusion. Recuperado de: $\quad$ http://www.oecd.org/daf/fin/financialeducation/2018- INFEFinLit-Measurement-Toolkit.pdf.

OCDE -Organización para la Cooperación y el Desarrollo Económicos (2020), PISA 2018 Results (Volume IV): Are Students Smart about Money? PISA, OECD Publishing, Paris, https://doi.org/10.1787/48ebd1ba-en.

Raccanello, K., \& Guzmán, E. H. (2014). Educación e inclusión financiera. Revista Latinoamericana de Estudios Educativos (México), 44(2), 119-141. Disponible en: https://www.redalyc.org/articulo.oa?id=270/2703126800 5

Valbuena Duarte, S., Marín-Tapia, K. A., \& De la Hoz, A. P. (2020). Desarrollo de competencias en educación económica y financiera para la toma de decisiones 
informadas del ciudadano común. Revista Logos Ciencia \& Tecnología, $12(1)$ 95109. http://dx.doi.org/10.22335/rlct.v12i1.1103. Vera, J. L. (2016). La (Des) educación Financiera en Jóvenes
Universitarios ecuatorianos: una aproximación teórica. Revista Empresarial, 10(37), 36-41. Recuperado de: https://dialnet.unirioja.es/servlet/articulo?codigo $=55803$ 38 